\title{
Application of Wireless Sensor Network to Make Transportation Fleet Information Systems Smart
}

\author{
ALI GHARAGOZLU ${ }^{1}$ and MEHDI IZADI ${ }^{2}$ \\ ${ }^{1}$ The B.S student of the Mechanical engineering in Bu-Ali Sina University, Hamadan, Iran \\ ${ }^{2}$ Faculty of electrical and computer engineering, University of Tabriz, Tabriz, Iran \\ E-mail: 'gharagozlu1390@gmail.com, ${ }^{2} m$-izadi91@ms.tabrizu.ac.ir
}

\begin{abstract}
Traffic signs are tools to control and regulate movement, on streets and crossings. These signs individually play their own essential role in reducing accidents and securing traffic. Unfortunately, thieves steal these signs on suitable occasions to sell them as scrape in black market. Wireless sensor systems are of desirable features including independence, being capable of expanding in remote and inconsistent places. They may be assigned such operations as supervising traffic signs. This essay aims at study of some hierarchical routing protocols or static cluster, introducing a new protocol through which we focus on clustering special nodes having no-battery in contrast to ordinary nodes. The result is to obtain network more stability and optimal energy consumption. The new protocol was tested on real characteristics of more than 600 traffic signs in the city of Hamedan, run in MTALAB software. The findings are examined here.
\end{abstract}

Keywords: Wireless Sensor Networks, Routing Protocols, Static Clustering, Optimal Energy Consumption, Life Cycle of the Network, MATLAB.

\section{INTRODUCTION}

To solve social and economic problems that the people around the world face, researchers try to, through such concepts as Smart City Week [1], reach novel approaches to these problems, using new technologies. Smart transportation is one of these efforts. In this regards, we may refer to the application of wireless networks and sensors in providing new urban functions for smart cities a new idea in communication of information in smart cities is digital signs [2].

\section{WIRELESS SENSOR NETWORKS}

WSN refer to a collection of sensors interconnected a wirelessly and all feel a specific phenomenon. The number of these sensors may be high and their distribution range may be vast. The sensors in these networks may be located manually or distributed randomly in places of interests to function. The main propose of these network, after establishment, is firstly to collects information and secondly to make the life cycle of system as longer as possible [3].

\subsection{Tracking Targets}

Tracking targets has recently attracted the attention of scientists in operational and research projects. Some of recently experimental projects in field of tracking and supervision are:

Underwater tracking and surveillance: Aqua nodes [4].

- Border surveillance: Exscal [5].

- System intrusion and recognition projects: line- in the - sand [6][7].

- Moving animals tracking in natural sources: zebraNet protests [8].

- Tracking using video camera: Mesh Eyes, Cyclops - Sens Eye [9][10][11].

The issue of tracking in WSN may be defined as a network of " $\mathrm{n}$ " wireless sensor nods formed to track targets in a region. "M" targets are located in this region. Sampling signals transmitted for 
targets (chemicals, vibrations, audio signals), these sensing nodes recognize them or their absence. In view of processing and formation of network structure, algorithms may be categorized into two distributed and concentrated ones. Under the latter method, one node (or a central entity) obtains information from the whole network (assuming that the transmission of information from all modes to this central mode). Then, the optimal structure (cluster of tree) is formed on the basis of cross-network data. By cluster-based method, a cluster is made of nodes before the setting up of the net (static 1) or at the same time as the target is recognized (dynamic)[12][13]. The group head (cluster head 3) is determined. The nodes send their data on the target is the related cluster head [14]. The cluster head locates the target after collection of data, sending the concerned report to sink node [15], based on tree method, similar to methods based on cluster, and before the start of network's operation or as soon as the recognition of target. The tree- structure is made of nodes; each mode sends its information to the parent node. The collection of data to the root node .The target is located and related report is sent by the root to the sink. under the hierarchical routing methods (based on clustering) $[16][17][18][19]$. The modes may process and transmit to more vigorously while nodes may be used to perform the sensing function in vicinity of the target, consuming low level of energy. In fact , the hierarchical method, forming clusters are allocating special duties to cluster heads, may greatly contribute to life cycle, energy efficiency of system and comparability, avoiding single cross architecture. The hierarchical routing is a basis for less energy demand of a cluster, through aggregation and combination of data to reduce the number of sent messages. Hierarchical routing was mostly two-layer routing, one of which is used to select the cluster head and the other is allocated the duty to routing. However, the most of technologies are not related to routing, they speak of who and when the process or aggregation of data, or allocation of channels [20].

\section{ROUTING PROTOCOL}

\section{$3.1 \mathrm{LEACH}$}

This protocol is one of the most famous hierarchical protocols for WSN (Figure 1) [21].

Under this protocol, the time is divided into equal turns. Each turn is divided into two phases. The second phase is related to the ordinary function of the network, named stable phase. In the first phase, based on comparison probability function $\mathrm{CH}$ is chose. (Figure2) .The choice of $\mathrm{CH}$ is made in this way that each sensing node chooses a number between one and zero in case this number is less than a fixed threshold. That node is chosen as $\mathrm{Ch}$ in that turn. This probability function has been selected in such a way that each mode is selected as Ch once per determined number turns.

By doing so, the consumption of energy is distributed over the net. Following the selection of $\mathrm{CHs}$ in the commissioning phase of each turn, each $\mathrm{CH}$ informs other modes of its selection. Each mode selects its appropriate $\mathrm{CH}$ for itself. This fact is notified to relate $\mathrm{CH}$. As result, clusters are formed.

At the next step, each $\mathrm{CH}$ provides it's clusters modes with time planning, allocating each sensor a phase to avoid the collision among data of sensors in each cluster [22].

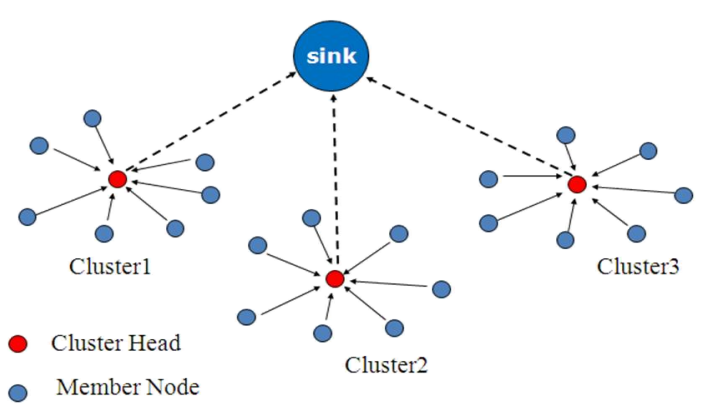

Fig. 1. LEACH protocol with cluster nodes

$$
\boldsymbol{T}(\boldsymbol{n})=\frac{P}{1-P *(\boldsymbol{r} \bmod 1 / P)} \text { if } \boldsymbol{n} \in \boldsymbol{G}
$$

Fig. 2. LEACH possibility of implementing

\subsection{SEP}

SEP is one of hierarchical protocols for wireless network, suitable for heterogeneous environments respect o the energy. We also assume that these sensors are not randomly distributed but the selection of cluster head are based on the imbalance of energy in the network [23].

\section{Solution:}

Selection of two parameters of PAdv and PNM. Each node knows all energy of the network, they respectively refer to the probability for being cluster head for normal and power - holding modes assuming.

\subsubsection{Properties of SEP}

- Its energy consumption is balanced 
- The probability for choosing power -holding modes to be chosen as cluster head is high.

- The life cycle of the net is high.

- It is comparable and dynamic.

n. $(1-m) \times P n r m+n . m$ padv $=n \times P o p t$

Fig. 3. The number of cluster heads in each round in heterogeneous environments

Averagely, the number of cluster head is chosen on the basis of the formula reflected in the (Figure $3)$.

$$
\begin{gathered}
\text { pnrm }=\frac{\text { Popt }}{1+\propto \cdot m} \\
\text { padv }=\frac{\text { Popt }}{1+\propto \cdot \mathrm{m}} \times(1+\propto)
\end{gathered}
$$

Fig. 4. Weight probability

The weight probability for ordinary and special nodes are padv and pnm respectively.

$$
\begin{gathered}
T(\text { Snrm })=\left\{\frac{\text { Pnrm }}{1-\text { Pnrm. }\left(r \bmod \frac{1}{\text { Pnrm }}\right)}\right. \\
T(\text { Sadv })=\left\{\frac{\text { Padv }}{1-\text { Padv } \cdot\left(r \bmod \frac{1}{\text { Padv }}\right)}\right. \\
0
\end{gathered}
$$

Fig.5 . Threshold

The selection of threshold for power -holding nods are reflected in (Figure 5).

\subsubsection{Comparison with LEACH}

- Complete consumption of additional energy in power-holding node.

- Longer life cycle is $26 \%$ higher than that of LEACH.

\section{NEW ROUTING PROTOCOL (SNN)}

All existing routing protocols under heterogeneous environment try to meet the balance in the selection of cluster head among special and ordinary cluster heads. This happens with the probability function of selecting in each group. This algorithm tries to take another probability in addition to previous cases. These probabilities are to take into consideration of special and ordinary clusters for candidate cluster heads from ordinary modes.

The algorithm of SNN selects the cluster head among ordinary nodes in line with (Formula 2).

S(i).ENERGY $\quad==0 \quad \& \& \quad$ booln $==$ true $\quad \& \&$ booln $2==$ true $\& \&$ temp_rand $<=($ pnrm*2 / $(1-$ pnrm * $\bmod (\mathrm{r}, \operatorname{round}(1 / \mathrm{pnrm}))))$

Formula 2

$\mathrm{S}(\mathrm{i})=$ number of arrange among ordinary nodes; booin $=$ Boolean variables.

(tem_rand) $=$ a numerical variable between 0 and 1 .

SNN chooses ordinary cluster head based on adjusting sensing range.

The adjustment of sensing range is a parameter based on which an ordinary node fixed the change to be selected as cluster head. This parameter should be of distance with less than meter between ordinary node of the candidate cluster head and specific cluster head . Also, this parameter should be in a distance less than one- meter between ordinary node (candidate cluster head) and ordinary one. Figures 4 and 5 show a sensing range.

\section{PROCESS OF DATA TRANSMISSION}

The data transmission may be performed 3 groups of nodes

1) Ordinary nodes

2) Ordinary cluster head

3) Special ( cluster head )

4) Ordinary nodes send their data to the neighboring cluster head node. The head may be either ordinary or special. 
A. Gharagozlu and M. Izadi / International Journal of Computer Networks and Communications Security, 2 (10), October 2014

In next section Figure 6 shows the distribution of these under the case study ordinary cluster nodes sends its own data or the data sent to it by a member to neighboring cluster to the special cluster head nodes in a shower manner. Figures 6 and 7 show the flow of data in WSN.

\section{IMPLEMENTATION}

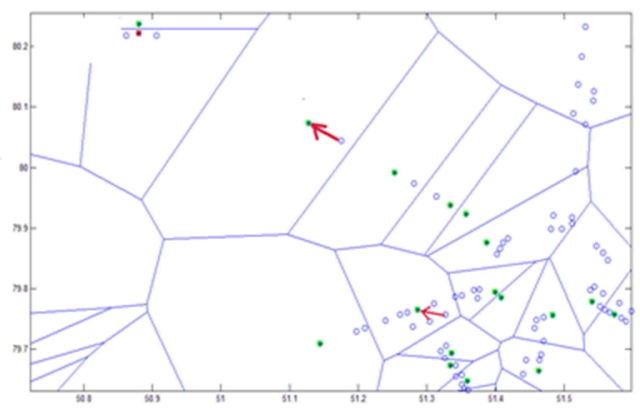

Fig. 6. Transmission of ordinary node with ordinary cluster head

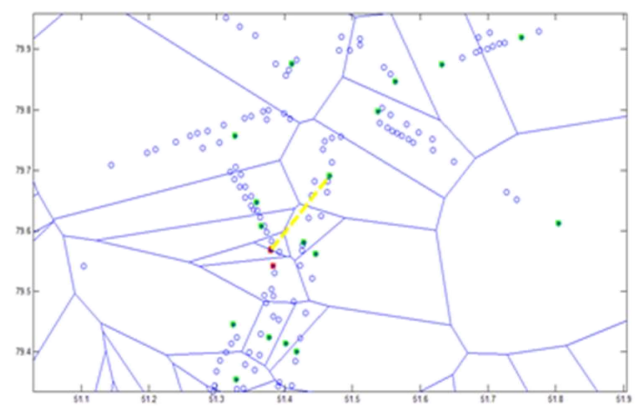

Fig. 7. find the nearest cluster head by the cluster-heads for ordinary

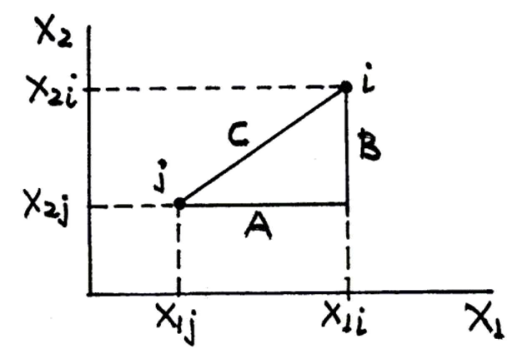

$D(i, j)=\sqrt{A^{2}+B^{2}}=\sqrt{\left(X_{1 i}-X_{1 j}\right)^{2}+\left(X_{2 i}-X_{2 j}\right)^{2}}$.

Formula 3 Calculate the Euclidian distance

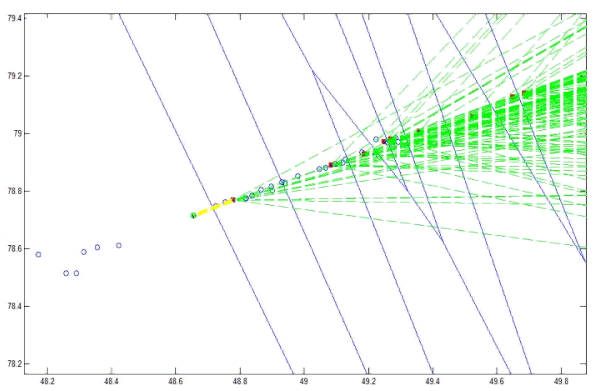

Fig. 8. Flooding

\subsection{Simulation}

SNN clustering sensing radius '1' of Figure 9 shows. Featured cluster head node is red and green cluster head nodes are normal.

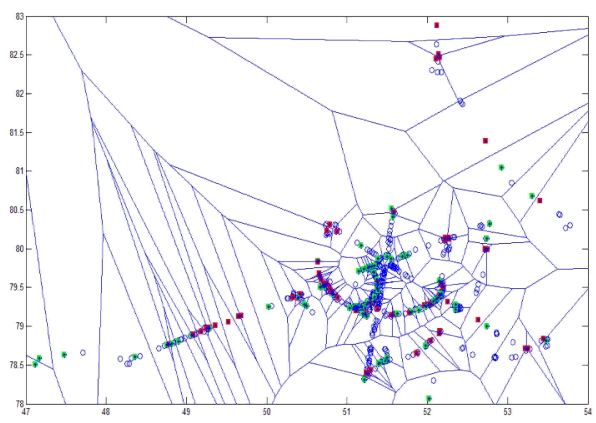

Fig. 9. sensing radius ' 1 '

SNN clustering sensing radius '4' of Figure 10 shows. Featured cluster head node is red and green cluster head nodes are normal.

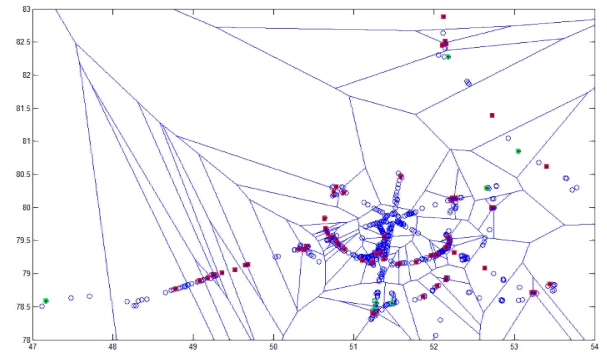

Fig. 10. sensing radius ' 4 '

SNN clustering sensing radius ' 5 ' of Figure 11 shows. Featured cluster head node is red and green cluster head nodes are normal. 


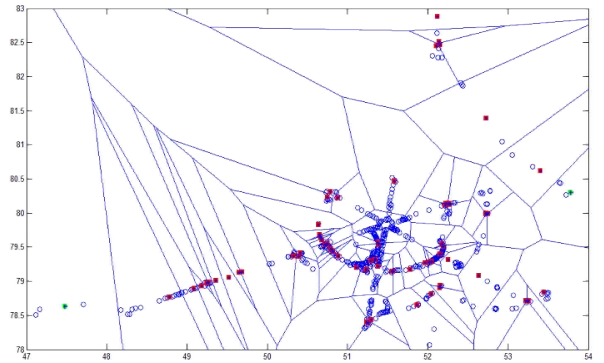

Figure 11 sensing radius ' 5 '

\subsection{Comparison of two methods for the selection of cluster heads energy consumption}

Figure12 compares the energy consumption patterns in the data by a typical cluster head node is the destination Sink. The red graph nodes and ordinary cluster head to the nearest cluster head node, especially for broadcast delivery. The blue Graphs has a normal cluster heads his own and delivers energy from the battery to the SINK. However, calculating the energy consumption among cluster heads of special flood the nature of the message protocol is compromise.

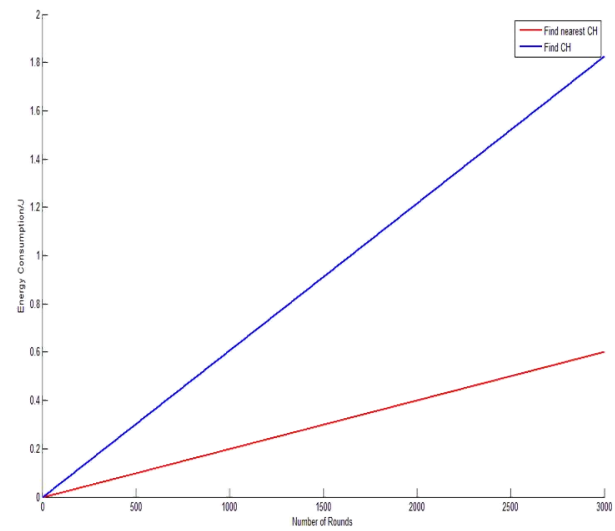

Fig. 12. compare the energy consumption associated with the cluster head

\subsection{The results of a comparison of three methods of energy SNN Clustering}

Figure 13 SNN protocol with three other protocols in terms of energy consumption shows.

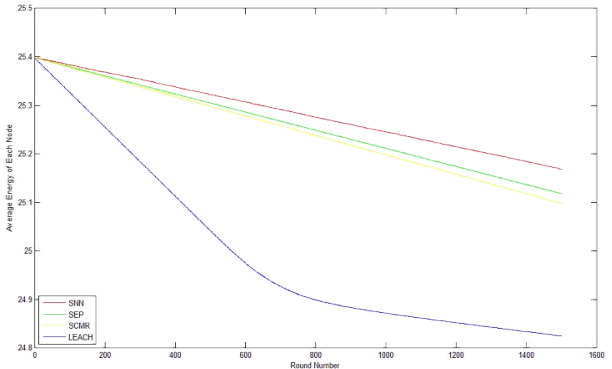

Fig. 13. compares the SNN with SCMR, SEP and LEACH in terms of power consumption displays

\subsection{Comparison of network lifetime SNN Clustering}

Figure 14 SNN protocol with three other protocols in terms of network lifetime show.

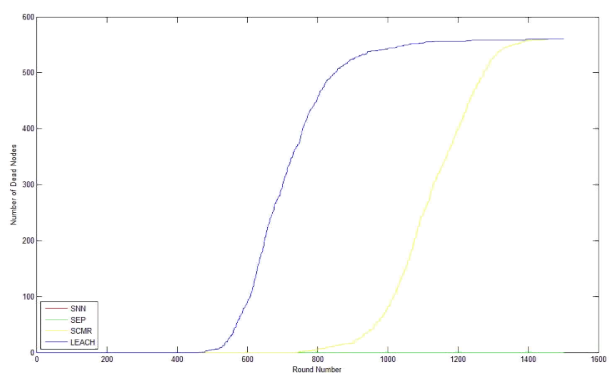

Fig. 14. compares the three protocols SNN SCMR SEP, and LEACH in terms of lifetime

\section{REFERENCES}

[1] Social Innovation 2014 / Smart City Week .2014 - October 29-31, 2014 in Yokohama, Japan

[2] Mateos, M.A.; Soto, P.R. ; Mezher, A.M."Smart city for VANETs using warning messages, traffic statistics and intelligent traffic lights"Dept. "Eng. Telematica., Univ. Politec. de Catalunya (UPC), Barcelona, Spain.

[3] Neha, J., "Energy Efficient And Cluster Based Routing Protocol For Wireless Sensor Network:A Review,". International Journal of Advance Technology \& Engineering Research, 2011. 1(1).

[4] I. Vasilescu, C. Detweiler, and D. Rus, "AquaNodes: An Underwater Sensor Network," Proc. Of ACM Int. Workshop on UnderWater Networks (WUWNet), Montreal, Quebec, Canada, September 2007, pp. 85-88. 
[5] A. Arora, R. Ramnath, E. Ertin, P. Sinha, S. Bapat, V. Naik, V. Kulathumani, H. Zhang, H. Cao, M. Sridharan, S. Kumar, N. Seddon, C. Anderson, T. Herman, N. Trivedi, C. Zhang, M. Nesterenko, R. Shah, S. Kulkarni, M. Aramugam, L. Wang, M. Gouda, Y. Choi, D. Culler, P. Dutta, C. Sharp, G. Tolle, M. Grimmer, B. Ferriera, and K. Parker, "ExScal: Elements of an Extreme Scale Wireless Sensor Network," Proc. of the 11th IEEE Int. Conf. on Embedded and Real-Time Computing Systems and Applications (RTCSA), Hong Kong, China, August 2005, pp. 102-108.

[6] A. Arora, P. Dutta, S. Bapat, V. Kulathumani, H. Zhang, V. Naik, V. Mittal, H. Cao, M. Demirbas,M. Gouda, Y. Choi, T. Herman, S. Kulkarni, U. Arumugam, M. Nesterenko, A. Vora, and M.Miyashita, "A line in the sand: a wireless sensor network for target detection, classification, and tracking," Computer Networks, vol. 46, pp. 605-634, July 2004.

[7] T. He, S. Krishnamurthy, L. Luo, T. Yan, L. Gu, R. Stoleru, G. Zhou, Q. Cao, P. Vicaire, J.A. Stankovic, T.F. Abdelzaher, J. Hui, B.H. Krogh, "VigilNet: An Integrated Sensor Network System for Energy Efficient Surveillance," ACM Trans. on Sensor Networks, vol. 2, no. 1., pp. 1-38., 2006.

[8] P. Zhang, C.M. Sadler, S.A. Lyon, and M. Martonosi, "Hardware design experiences in ZebraNet," Proc. of the SenSys'04, Baltimore, MD, 2004.

[9] S. Hengstler, D. Prashanth, S. Fong, and H. Aghajan, "MeshEye: A Hybrid-Resolution Smart Camera Mote for Applications in Distributed Intelligent Surveillance," Proc. of IPSN, Cambridge,Massachusetts, USA, April 2007, pp. 360-369.

[10] P. Kulkarni, D. Ganesan, P. Shenoy, and Q. Lu, "SensEye: A Multitier Camera Sensor Network,"Proc. of the 13th Annual ACM Int. Conf. on Multimedia (MM), Singapore, November, 2005, pp.229-238.

[11]M. Rahimi, R. Baer, O.I. Iroezi, J.C. Garcia, J. Warrior, D. Estrin, and M. Srivastava, "Cyclops: in situ image sensing and interpretation in wireless sensor networks," Proc. of the 3rd Int. Conf. on Embedded Networked Sensor Systems (Sensys), San Diego, CA, 2005, pp. 192-204.

[12]Bakht, H.,"Routing protocols for mobile ad honetworks", GERI Annual Research Symposium .(GARS) Liverpool, UK, June, $2005 \mathrm{c}$

[13]I. F. Akyildiz et al., "Wireless Sensor Networks: A Survey," Elsevier Sci. B.V.
Comp. Networks, vol. 38, no. 4, Mar. 2002, pp. 393-422.

[14] Gholam Kosar, M.D., VOIP in Ad hoc Network, King Fahd University of Petroleom and Minerals, Saudi Arabia,pp. 22-26, 2006.

[15]F. Simonota "Real-time communications using TDMA-based multi-access protocol, Y.Q. SongComputer Communications 20 (1997) 435-.448

[16] Heinzelman, W., A. Chandrakasan, and Balakrishnan. Energy Efficient Communication Protocol for Wireless Microsensor Netwroks (LEACH). In Proceedings of 33rd Hawaii international conference systems science. 2004.

[17] Qing, L., Q. Zhu, and etc, "Design of a distributed energy-efficient clustering algorithm for heterogeneous wireless sensor networks". Computer Communications, August, 2006. 29(12): p. 2230-2237.

[18][18] Manjeshwar, A. and D.P. Agarwal, "TEEN: a routing protocol for enhanced efficiency inwireless sensor networks", in 1st InternationalWorkshop on Parallel and DistributedComputing Issues in WirelessNetworks and Mobile Computing. April 2001.

[19] Smaragdakis, G., I. Matta, and A. Bestavros, "SEP: A stable election protocol for clustered heterogeneous wireless sensor networks".Boston University Computer Science Department, 2004.

[20]D. Chen and P.K. Varshney, "QoS Support in Wireless Sensor Networks: A Survey," Proc. of the 2004 Int. Conf. on Wireless Networks (ICWN), Las Vegas, Nevada, June 2004, pp. 227-233.

[21] Heinzelman, W., A. Chandrakasan, and HBalakrishnan. Energy Efficient Communication Protocol for Wireless Microsensor Netwroks (LEACH). In Proceedings of 33rd Hawaii international conference systems science. 2004.

[22] Wendi B. Heinzelman, Member, IEEE, Anantha P. Chandrakasan, Senior Member, IEEE, and Hari Balakrishnan, Member, IEEE"An Application-Specific Protocol Architecture for Wireless Microsensor Networks",IEEE TRANSACTIONS ON WIRELESS COMMUNICATIONS, VOL. 1, NO. 4, OCTOBER 2002. 